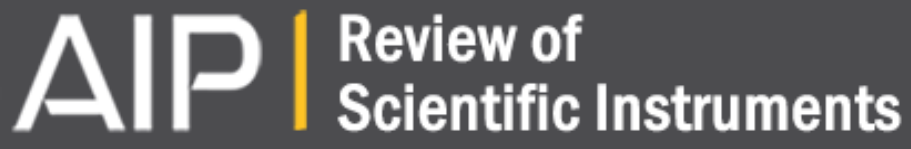

Smart monitoring system based on adaptive current control for superconducting cable test

Pasquale Arpaia, Amalia Ballarino, Vincenzo Daponte, Giuseppe Montenero, and Cesare Svelto

Citation: Review of Scientific Instruments 85, 125111 (2014); doi: 10.1063/1.4902977

View online: http://dx.doi.org/10.1063/1.4902977

View Table of Contents: http://scitation.aip.org/content/aip/journal/rsi/85/12?ver=pdfcov

Published by the AIP Publishing

\section{Articles you may be interested in}

New methodology of speed-control of photovoltaic pumping system

J. Renewable Sustainable Energy 5, 053109 (2013); 10.1063/1.4821213

Fuzzy logic control of wind energy conversion system

J. Renewable Sustainable Energy 5, 023125 (2013); 10.1063/1.4798739

Performance improvement of a measurement station for superconducting cable test

Rev. Sci. Instrum. 83, 095111 (2012); 10.1063/1.4752410

Manufacturing of $50 \mathrm{kA}$ superconducting transformer for ITER correction coil conductor test

Rev. Sci. Instrum. 81, 044701 (2010); 10.1063/1.3382346

A superconducting transformer system for high current cable testing

Rev. Sci. Instrum. 81, 035107 (2010); 10.1063/1.3340888

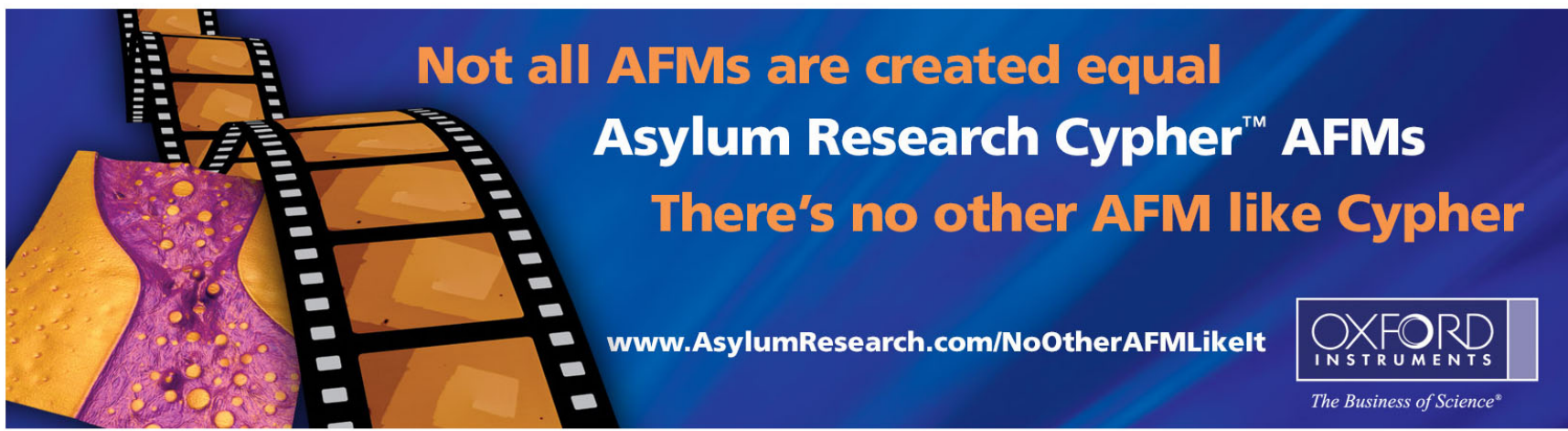




\title{
Smart monitoring system based on adaptive current control for superconducting cable test
}

\author{
Pasquale Arpaia, ${ }^{1,2}$ Amalia Ballarino, ${ }^{2}$ Vincenzo Daponte, ${ }^{2,3}$ Giuseppe Montenero, ${ }^{2}$ \\ and Cesare Svelto ${ }^{3}$ \\ ${ }^{1}$ Department of Electrical Engineering and Information Technology, University of Naples Federico II, \\ 80125 Napoli, Italy \\ ${ }^{2}$ Technology Department, European Organization for Nuclear Research (CERN), 1217 Geneva, Switzerland \\ ${ }^{3}$ Department of Electronics, Information, and Bioengineering, Polytechnic of Milan, 20133 Milano, Italy
}

(Received 11 July 2014; accepted 18 November 2014; published online 12 December 2014)

\begin{abstract}
A smart monitoring system for superconducting cable test is proposed with an adaptive current control of a superconducting transformer secondary. The design, based on Fuzzy Gain Scheduling, allows the controller parameters to adapt continuously, and finely, to the working variations arising from transformer nonlinear dynamics. The control system is integrated in a fully digital control loop, with all the related benefits, i.e., high noise rejection, ease of implementation/modification, and so on. In particular, an accurate model of the system, controlled by a Fuzzy Gain Scheduler of the superconducting transformer, was achieved by an experimental campaign through the working domain at several current ramp rates. The model performance was characterized by simulation, under all the main operating conditions, in order to guide the controller design. Finally, the proposed monitoring system was experimentally validated at European Organization for Nuclear Research (CERN) in comparison to the state-of-the-art control system [P. Arpaia, L. Bottura, G. Montenero, and S. Le Naour, "Performance improvement of a measurement station for superconducting cable test," Rev. Sci. Instrum. 83, 095111 (2012)] of the Facility for the Research on Superconducting Cables, achieving a significant performance improvement: a reduction in the system overshoot by $50 \%$, with a related attenuation of the corresponding dynamic residual error (both absolute and RMS) up to 52\%. (C) 2014 AIP Publishing LLC. [http://dx.doi.org/10.1063/1.4902977]
\end{abstract}

\section{INTRODUCTION}

Superconductivity is used in several electrical applications related to basic research and experimental physics. For those applications requiring high-current cables, a specific characterization of the superconducting cable properties is a key step of the development. In particular, measurement and hence knowledge of the critical current at nominal operating temperature and field plays a fundamental role. ${ }^{2}$ The critical current at a given temperature and field defines the upper working condition where the cable looses its superconducting property, taking on a resistive behavior. Under this circumstance, particular attention has to be paid to proper discharge of the residual current in the circuit under test. This system commonly involves current levels in the order of the tens of $\mathrm{kA}$, to be properly dissipated.

At the European Organization for Nuclear Research (CERN), superconductivity has been studied and used for many years and it plays an essential role in the particle accelerator Large Hadron Collider (LHC). In this regard, within the activities of research and development of superconducting magnets, a Facility for the Research on Superconducting Cables (FReSCa) ${ }^{3}$ was specifically built. This facility is still used today for the characterization of high-current cables made from novel advanced superconductors. In FReSCa, the cables are tested to assess their properties experimentally, in particular the critical current. Current is driven in the sample cable under test through a power converter operated at room temperature. ${ }^{4}$ This configuration, although allowing high cur- rents to be produced at high ramp rates, results expensive both in terms of electric power dissipation and liquid helium consumption. A 70-kA superconducting transformer, hosted in the same cryostat with the cable under test, was introduced to overcome these issues. This allows a significantly higher current to be induced in the transformer secondary (where the sample cable is placed) by a relatively lower primary current (a few tens of ampere). ${ }^{5,6}$ The current at the primary of the transformer is provided through a low-power voltagecontrolled current source, drastically reducing the consumption of both electric power and helium boil off. However, for the measurement of current at cryogenic temperatures, the superconducting transformer presents the challenging issue imposed by the need for an accurate control: ${ }^{7}$ a few percent accuracy of the current levels is required. The available current control strategy, even suitable for the task of cable testing, operates under several constraints mainly due to the transformer nonlinear behavior. ${ }^{1}$

The ideal transfer function of an air-core superconducting transformer can be written as ${ }^{1}$

$$
\begin{aligned}
H_{T}(s) & =\frac{I_{s}(s)}{I_{p}(s)}=G_{T} \frac{s}{s+\frac{1}{\tau}} \\
\text { with } \quad G_{T} & =M /\left(R_{\mathrm{s}} \tau\right) \text { and } \tau=\left(L_{\mathrm{s}}+L_{\mathrm{sp}}\right) / R_{\mathrm{s}} \text {, }
\end{aligned}
$$

where $G_{T}$ is the transformer gain, $\tau$ is the time constant, $M$ is the mutual coupling between primary and secondary, $R_{\mathrm{s}}$ is the 

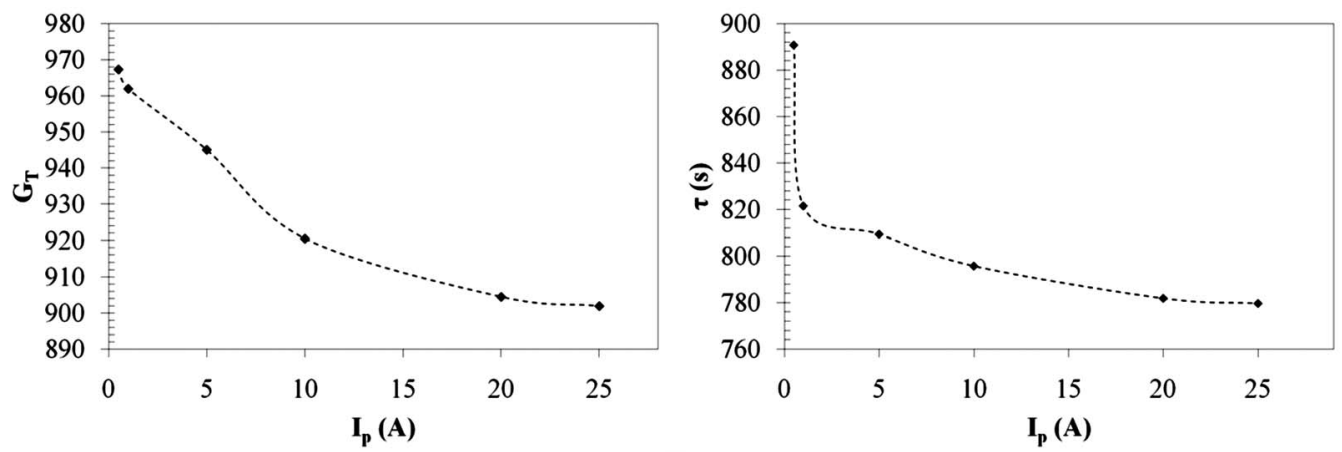

FIG. 1. Transformer gain $G_{T}$ (left) and decay time constant $\tau$ (right) as a function of the maximum current reached on the primary $I_{p}$.

total resistance of the secondary, $L_{\mathrm{s}}$ is the inductance of the secondary, and $L_{\mathrm{sp}}$ is the inductance of the sample.

However, the electrical parameters of the transformer such as the resistance of the joint connecting the cable under test, the transformer secondary, and the self-inductance, depend on both the current and the field. ${ }^{8,9}$ Moreover, the sample inductance can differ from its nominal value. ${ }^{10}$ These effects lead the parameters of the controlled circuit to deviate from their theoretical values, giving rise to a nonlinear behavior to be compensated by the control system. In Fig. 1, as an example, the measured gain (left) and the time constant (right) of) for the 36-kA superconducting transformer, in a short-circuit configuration, available at the CERN FReSCa test station ${ }^{1}$ are shown as a function of the maximum primary current $I_{p}$. Those parameters deviate from their ideal values $G_{T}=877$ and $\tau=1000 \mathrm{~s}$, for $M=8.77 \mathrm{mH}$, $R_{s}=10 \mathrm{n} \Omega, L_{s}=9 \mu \mathrm{H}$, and $L_{s p}=1 \mu \mathrm{H}$, due to the powering conditions, namely, current levels translating into field dependence too.

The available controller configuration does not allow sudden accelerations in the induced current, especially if followed by steep ramps. Thus, an ad hoc setup for the test reference curves is needed to operate within the controller boundaries.

In this work, the test current performance is improved with respect to the state-of-the-art controllers by using an adaptive control strategy ${ }^{8}$ integrated in the measurement station real-time monitoring. The current is driven in the transformer secondary, thus in the cable under test, by continuously adapting the control logic to the working condition variations arising from the transformer nonlinear dynamics. This algorithm follows the principle of the Fuzzy Gain Scheduling 9 and manages the transitions between two different system conditions through a fuzzification of the main state variables. ${ }^{10}$ In Sec. II, a background on the basic principles underlying the control system is given. In Sec. III, the proposed control system is outlined and, in Sec. IV, experimental results from the on-field characterization and validation at CERN in the FReSCa laboratory are illustrated.

\section{BACKGROUND}

In the following, a background on the principles of (A) the Gain Scheduling adaptive control and (B) the Fuzzy logic techniques is provided.

\section{A. The gain scheduling adaptive control}

Gain Scheduling (GS) is one of the most popular approaches to nonlinear control design and has been widely applied in various fields such as aerospace or process control. ${ }^{11}$ This divide-and-conquer approach is the main reason of the popularity of gain scheduling methods, since it enables the well-established linear design methods to be applied to nonlinear problems.

The key idea of Gain Scheduling consists on finding rules between the changes of the model and significant variables (Fig. 2), hence the feedback is nonlinear and it may be implemented as a look-up table. Thus, a look-up table with an appropriate logic for detecting the operating point and choosing the corresponding controller values is needed.

The advantage of gain scheduling is that the controller parameters can be changed as quickly as the auxiliary measurements respond to parameter changes. Frequent and rapid changes of the controller gains, on another side, may lead to instability; therefore, a limit has to be placed on how often and how fast the controller gains can be changed. An incorrect schedule caused by the lack of a compensating feedback, together with the unpredictable changes in the plant dynamics, may lead to performance worsening or even to complete failure of the control system.

A drawback of the Gain Scheduling, usually less significant for complex and already costly systems, is the increase of the design and implementation costs according to the number of operating points.

Another attractive approach for wide-range control of nonlinear processes is the Multimode control ${ }^{10}$ technique. In this method, a switching among several controllers, each

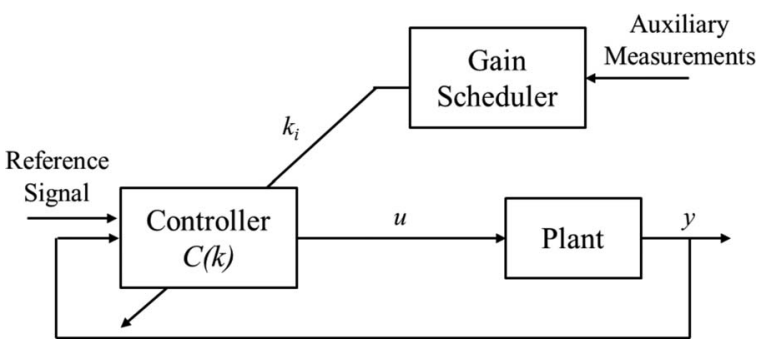

FIG. 2. Gain scheduling general scheme. 


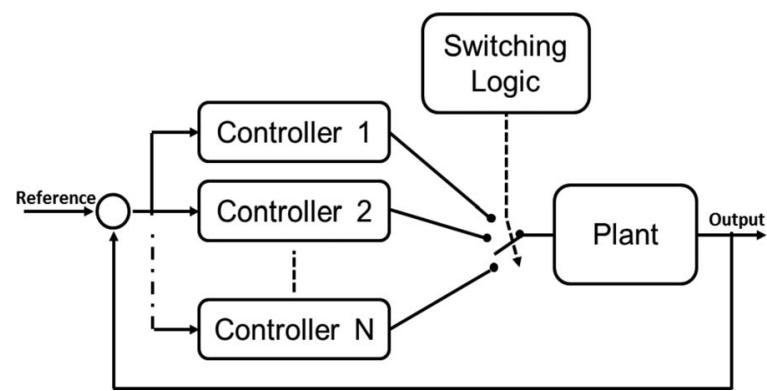

FIG. 3. Multimode control scheme.

designed for a partition of the operating space, is made in accordance to the operating conditions (Fig. 3).

This approach is equivalent to the Gain Scheduling provided the controllers to have the same structure. In general, however, the controllers do not need to have the same structure. This feature allows great flexibility in the type of control techniques that can be adopted. The major concern is to achieve bump-less transfer between two adjacent controllers when the switching occurs and to avoid intermittent operation of two controllers in adjacent operating regions with sharp boundaries. In this method, as well as for the Gain Scheduling, the implementation of the interpolation strategy and the switching logic is a key issue to achieve a satisfactory behavior in a wide operating space.

The large variety of domains and applications where Fuzzy GS-based strategies were exploited suggests their adaptability to different kinds of instrumentation and measurement processes with some common peculiar aspects. Most of the problems addressed in literature are characterized by a wide operation domain. ${ }^{11,12,14,15}$ In monitoring systems for superconducting cable test, typical operating ranges are large, like the span of $32 \mathrm{kA}$ of the test facility at CERN. A further peculiar aspect is the nonlinearity of the systems to be monitored arising from physical phenomena included in the systems dynamics. Often the nonlinearities affect the system behavior differently in the diverse regions of the operating domain. In most cases, designing an ad hoc strategy turns out to be more effective to compensate the behavior in these regions rather than tuning a unique strategy on the domain, as a whole. Therefore, when these aspects of wide operating range, nonlinear behavior, and significantly different nonlinearity impact in the specific range occur simultaneously, the use of a Fuzzy Gain scheduling becomes precious.

The design of a GS-based controller typically proceeds in several steps. In a first step, a variable $\alpha$, strongly correlated with the changes in the process dynamics, has to be chosen as the scheduling variable. This variable should be readily available and its time dependency easily manageable. In a second step, a set of operating points covering the whole operating range of the process should be identified. This set defines a vector of values, $A=\left\{\alpha_{1}, \ldots, \alpha_{n}\right\}$, in the scheduling variable and a partitions of the operating space. In a third step, the linear controller at each operating point has to be designed, using the linear time-invariant models at those points if required, and set the controller parameters. Finally, the gain scheduling interpolating scheme has to be delineated, to al- low the controller parameters being selected according to the corresponding operating points.

Given excellent robust local stability and performance properties at the selected operating points, there is no guarantee that these properties will hold at all points and between them. This possibility is highlighted by the local nature of the control methods even if they are theoretically well-supported in a real application. Thus, in general, and not exclusively for Gain Scheduling, the control system properties are validated through extensive computer simulation experiments.

\section{B. Fuzzy logic techniques}

Fuzzy systems are nonlinear modeling paradigms, ${ }^{12}$ robust with respect to noise in the data. Representing problems in a simple and clear way is a typical capability of the fuzzy systems. They also are able to perform reasoning on inaccurate information and to give a clear representation of the evolution of the model providing understandable explanations about the model progression.

The Takagi-Sugeno-Kan (TSK) inference approach ${ }^{13}$ is widely used in control systems, in this model the rules are in the form:

$$
\begin{array}{r}
\mathrm{R}_{1}: \operatorname{IF}\left(x_{1} \text { is } A_{11}\right) \wedge\left(x_{2} \text { is } A_{12}\right) \wedge \ldots \wedge\left(x_{l} \text { is } A_{1 l}\right) \operatorname{THEN} y=z_{1}, \\
\mathrm{R}_{2}: \operatorname{IF}\left(x_{1} \text { is } A_{21}\right) \wedge\left(x_{2} \text { is } A_{22}\right) \wedge \ldots \wedge\left(x_{l} \text { is } A_{2 l}\right) \operatorname{THEN} y=z_{2}, \\
\ldots \\
\mathrm{R}_{j}: \operatorname{IF}\left(x_{1} \text { is } A_{j 1}\right) \wedge\left(x_{2} \text { is } A_{j 2}\right) \wedge \ldots \wedge\left(x_{l} \text { is } A_{j l}\right) \text { THEN } y=z_{j},
\end{array}
$$

where

- $x_{1}, x_{2}, \ldots, x_{l}$ are the system input variables;

- $A_{j i}$ are the possible fuzzy set describing the input variables (each of them is associated to its own membership function);

- The antecedent of each rule is made up by a conjunction (" $A N D$ " operator) of propositional clauses (" $x$ is A");

- $y$ is the output variable and it can assume only crisp values $z_{j}$ (i.e., numerical).

A system including such rules can be represented by means of a nonlinear function of the input variables:

$$
y=f\left(x_{1}, x_{2}, \ldots, x_{l}\right) .
$$

The crisp value of the output variable is assessed by taking into account all the rules and their activation values $a$, with respect to the $z_{j}$ value assumed by the $y$ variable in each rule:

$$
y=\frac{z_{1} \cdot a\left(R_{1}\right)+z_{2} \cdot a\left(R_{2}\right)+\cdots+z_{j} \cdot a\left(R_{j}\right)}{a\left(R_{1}\right)+a\left(R_{2}\right)+\cdots+a\left(R_{j}\right)} .
$$

In summary, the activation level of the $j$ th rule is expressed by the "Larsen Product" operator or by any other $T$-norm derivable for the "AND" operator:

$$
a_{j}=\prod_{i=1}^{l} A_{j i}\left(x_{i}\right) .
$$

Equation (2.3) shows how to calculate the activation level of the $j$ th rule with the Larsen product. The system output is 
(a)

(b)

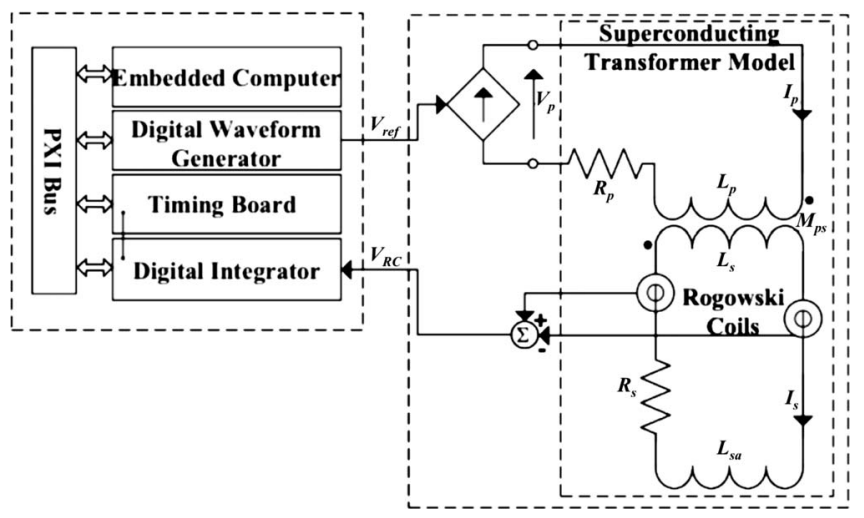

FIG. 4. Architecture of (a) measurement and control system and (b) system under control. ${ }^{1}$

then the centre of gravity of the local outputs:

$$
y=\frac{\sum_{j} a_{j} z_{j}}{\sum_{j} a_{j}} .
$$

\section{PROPOSAL}

In this section, (A) the architecture, (B) the model identification, and (C) the controller design of the novel smart monitoring system based on adaptive current control for superconducting cable test are outlined.

\section{A. Architecture}

During the development of the enhanced current control of FReSCa at CERN (Fig. 4), ${ }^{1}$ the system under control (Fig. 4(b)) showed a nonlinear behavior. In the development of its control strategy, a linearized model based on the ideal transformer physical equations was considered. As a result, a digital proportional integral (PI) controller operating within certain limitations was implemented. In order to obtain a significant performance improvement, a controller able to operate beyond the currently imposed limits, i.e., the start and stop current ramping acceleration of $800 \mathrm{~A} / \mathrm{s}^{2}$, is needed. To this aim, in the design process, a more detailed model taking into account nonlinear dynamics of the system under control is to be developed for its entire operating domain. The system to be controlled is composed by the cascade of the power supplier and the superconducting transformer (Fig. 4(b)).

The architecture of the smart monitoring system depicted in Fig. 5 aims at overcoming the drawbacks shown by the

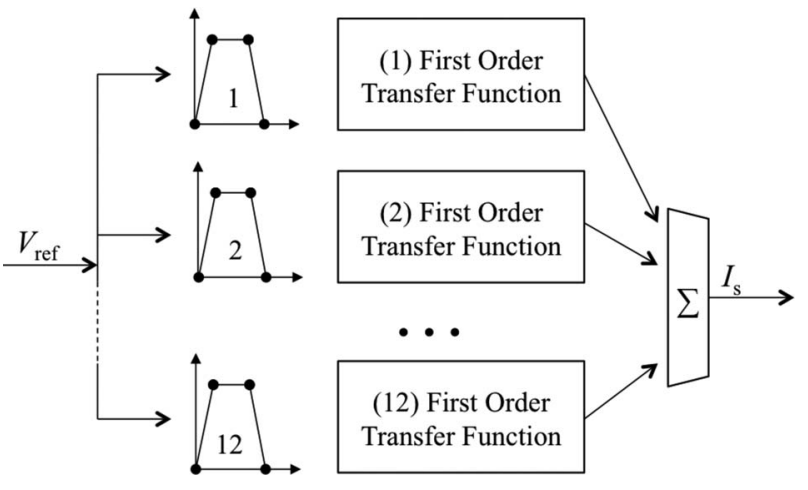

FIG. 6. Simulated model of the system under control.

state-of-the-art system, ${ }^{1}$ related to the transformer secondary current control, such as the limited bandwidth, the difficulties for the operator to set up a current cycle and the controller parameters tuning depending on the working conditions. ${ }^{1}$

The system input is the electrical current reference $I_{\text {ref }}$ to be fed into the superconducting cable while the output is the current $I_{\mathrm{s}}$ measured on the transformer secondary. The field of the nonlinear systems identification is wide and the included techniques can be either mathematical or statistical inferencebased. ${ }^{14}$ The physical analysis, of the magnetic couplings and of the other physical phenomena acting in the cryogenic part of the superconducting transformer, is extremely complex. Therefore, it would be a highly difficult task to build a model based on a sufficiently detailed physical analysis; therefore, an inferential approach was adopted to define the model.

\section{B. Model identification}

An exhaustive characterization of the system inputoutput dynamics was obtained by carrying out an extensive measurement campaign in several different working conditions. The system dynamic, for each working condition is identified independently from the ramp rate; in particular, the function defined for all the 12 regions identified approximates the behavior of the system for each considered ramp rate. At the end of this analysis, in order to synthesize a single model of the entire system domain (Fig. 6), the various functions have been included into a TSK fuzzy system. ${ }^{13}$

This fuzzy system approximates the system dynamics by scheduling each subdomain transformer function output according to the system input variations. The fuzzy system input variable is the power supply input voltage $V_{\text {ref }}$, the same

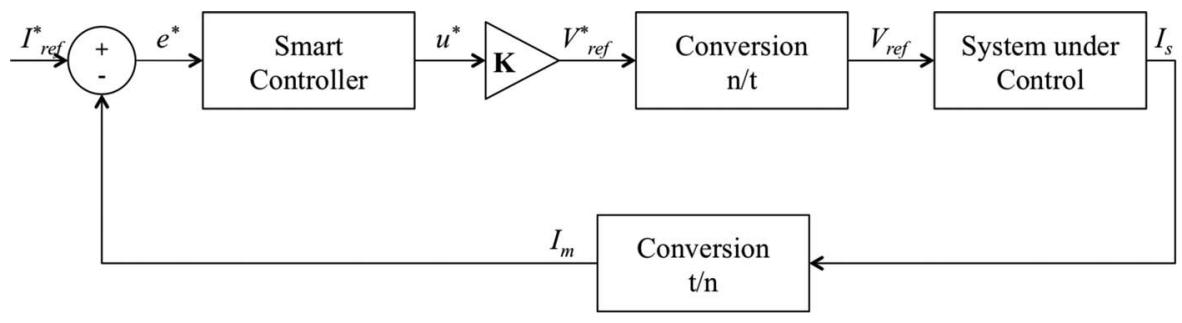

FIG. 5. Architecture of the novel smart monitoring based on adaptive current control. 


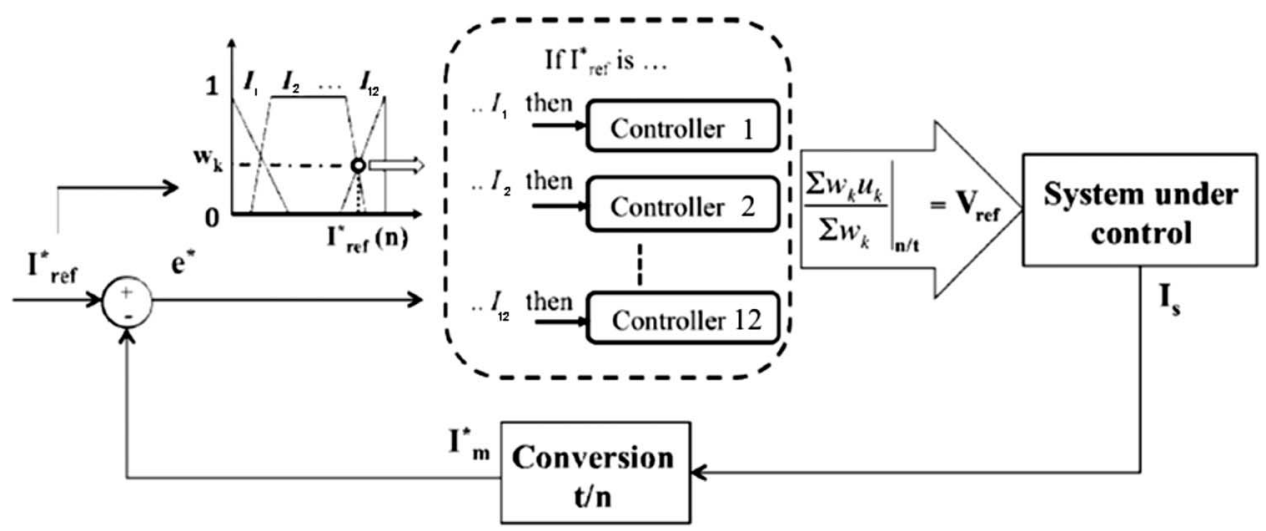

FIG. 7. PI-FGS system with one input divided into 12-subspaces.

of the system to be controlled. The domain of this variable is fuzzified by taking into account the operating regions identified by looking at the secondary current measurements. The shape of the membership functions is trapezoidal in order to have a clear definition of the subdomain mapped. The consequent of the TSK system rules is represented by the computed transfer functions. Each of them is characterized by a secondorder denominator and a first-order numerator. Each rule, one for each membership function, has the same unitary weight. The output of the simulated system is given by the sum of the outputs of each consequent, weighed with the truth value of the corresponding membership function.

\section{Controller design}

Fuzzy Gain Scheduling with PI controller (PI-FGS) is a particular implementation of the Gain Scheduling. In this scheme, the system operating space is divided into a number of subspaces or partitions, assigning a generalized PI controller to each one of them. Each controller is tuned taking into account the plant dynamics in its partition. The main feature of PI-FGS resides in the use of Fuzzy logic as switching logic and the interpolation or GS function. ${ }^{15}$ Fuzzy inference is used to implements the mechanism in order to detect the plant current operating conditions. Inference rules implement the generalized PI local controllers, one per rule (Fig. 7). The PI-FGS controller is based on a TSK fuzzy system ${ }^{13}$ with two inputs and one output.

The first input enters the scheduling variable $\alpha$, which is identified as the current $I_{\text {ref }}$ fed on the transformer secondary. The other input is the error signal $e^{*}(k)$, defined as the distance between the plant output $I^{*}{ }_{m}(k-1)$ and the desired one $I^{*}{ }_{\text {ref }}(k)$, required by the generalized PI to calculate the control signal (Fig. 8). The output of the TSK fuzzy system is the

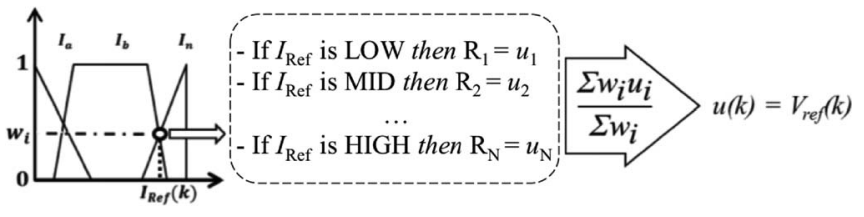

FIG. 8. FGS with PI controllers implemented in the TSK rule base. The rules $(\mathrm{R} 1, \mathrm{R} 2, \ldots, \mathrm{RN})$ are in the same form as formula (3.1). control signal $u(k)$ that, in the superconducting transformers system is the power supply voltage input $V_{\text {ref }}$.

The TSK fuzzy system has the following main characteristics: (i) the scheduling variable membership functions are trapezoidal and (ii) a singleton fuzzification method is used to simplify calculations by the inference mechanism.

The inference system relies on a rule base with individual rules. The total output $u(k)$ is the weighted average combination of all rule outputs. Rules have the form,

$$
\begin{aligned}
\operatorname{IF} \alpha \text { is } A_{i} \operatorname{THEN} u_{i}(k)= & K_{i} \cdot V_{\text {ref }}(k-1)-K_{i} \cdot K_{i I} \cdot y(k-1) \\
& +K_{i} \cdot K_{i P} \cdot e(k),
\end{aligned}
$$

where $i \in[1, \mathrm{R}]$ is the rule number, $A_{i}$ is the fuzzy set defining the $i$ th partition of the operating space, $K_{i P}, K_{i I}$, and $K_{i}$ are the generalized PI parameters or gains of the $i$ th rule or controller, and $u_{i}(k)$ is the control signal generated by the $i$ th rule or controller. The total control signal, generated by the TSK fuzzy system, is the weighted average of the control signals generated by each rule or controller,

$$
u(k)=\Sigma^{\mathrm{R}} w_{i} u_{i}(k) / \Sigma^{\mathrm{R}} w_{i}
$$

where the weights $w_{i}$ are the product of the membership values of the inputs being fuzzified. Since only the first input is being fuzzified,

$$
w_{i}=\mu A_{i}(\alpha)
$$

From (3.1)-(3.3), the control signal change $u(k)$ is

$$
\begin{aligned}
u(k)= & \Sigma_{i}^{R} \mu A_{i}(\alpha) \cdot\left(K_{i} \cdot V_{\text {ref }}(k-1)-K_{i} \cdot K_{i I} \cdot y(k-1)\right. \\
& \left.+K_{i} \cdot K_{i P} \cdot e(k)\right) / \Sigma_{i}^{R} \mu A_{i}(\alpha) .
\end{aligned}
$$

This is the PI-FGS controller output for the plant: in the system under exam it would be the voltage value $V_{\text {ref }}$ given to the power supply. At the end of the design process, the $C++$ implementation of the PI-FGS controller was completed with the parameters computed by simulating the controller with the computed system model. 


\section{EXPERIMENTAL RESULTS}

The effectiveness of the proposed smart monitoring, in providing a tight control of the transformer secondary current, was demonstrated experimentally on the superconducting transformer of $\mathrm{FReSCa}^{3}$ at CERN. In particular, main validation aim was to prove the PI-FGS algorithm capability of improving dynamic performance without loosing in metrological performance. In measurement stations for superconducting cable testing, among all the step response parameters, dynamic performance is assessed usually in terms of overshoot and the related attenuation of the corresponding dynamic residual error.

In the following, (A) the experimental setup, (B) the preliminary assessment, and $(\mathrm{C})$ the validation results from the on-field characterization are illustrated.

\section{A. Experimental setup}

According to the architecture of Fig. 5, the measurement and control setup was implemented as shown in Fig. 9. The waveform generator is realized through a data acquisition board NI-PXI 6281 by National Instruments. ${ }^{16}$

This multifunctional board also provides digital I/O, for interfacing the standard FReSCa quench protection and data acquisition systems, ${ }^{17}$ and analog inputs in the range of $\pm 1 \mathrm{~V}$, with a resolution of $30 \mu \mathrm{V}$, for the system characterization. The board drives a four-quadrants power supply Lake Shore Model 622 (ratings $\pm 100 \mathrm{~A}, \pm 5 \mathrm{~V}$ ), ${ }^{18}$ supplying the transformer. The nominal gain of the power supply is $\mathrm{GPS}=100 \mathrm{~A} / \mathrm{V}$ for an input control voltage range of $\pm 1 \mathrm{~V}$.

The core of the system is the Fast Digital Integrator $(\mathrm{FDI})^{19}$ for the transformer's secondary current measurements via the signal from the Rogowski coils. The signal-to-noise and distortion ratio is higher than $100 \mathrm{~dB}$. Typical static nonlinearity, relative to a full scale of $\pm 10 \mathrm{Vs}$ and with temperature ranging between 27 and $35^{\circ} \mathrm{C}$, is within $\pm 7 \mathrm{ppm}$.

The transfer function has typical relative errors of $0.2 \%$ (without specific calibration) for the gain and $17 \mathrm{ppm}$ (on the full scale) for the offset. Typical stability is $\pm 1 \mathrm{ppm}$ over $30 \mathrm{~min}$ at $30^{\circ} \mathrm{C}$. The timing board is a NI PXI-6682 by National Instruments, with a $10 \mathrm{MHz}$ internal clock, ${ }^{20}$ used to generate the trigger signal for the FDI and for the data acquisition board.

The boards are housed in a PXI crate U1091AC50 by Agilent. ${ }^{21}$ The embedded computer is a Single-Board Computer D9-6U by Mikro Elektronik, ${ }^{22}$ hosting the software handling the overall system functions, based on the Flexible Framework for Magnetic Measurements. ${ }^{23}$

\section{B. Preliminary assessment}

For the PI-FGS control system, performance was assessed by using the same setup of the reference control system characterization, ${ }^{1}$ that is, the insert containing the transformer is closed on a short circuit. The adaptive control was then validated by verifying the compatibility of critical current measurements with the results collected exploiting the reference control system (i.e., PI strategy-based). The superconducting cable for those measurements is the same used for the validation of the PI-based system vs. the 32-kA Power Supply system, namely an LHC cable of type $2,{ }^{24}$ that is, a keystone cable $\left(0.90^{\circ}\right)$ with 36 strands of $0.825 \mathrm{~mm}$ diameter (critical current at $6 \mathrm{~T}$ better than $12 \mathrm{kA}$ at $1.9 \mathrm{~K}^{4}$ ).

The preliminary tuning phase of the PI-FGS system has the aim of understanding performance limit in terms of reference tracking and eventually corrects controller's parameters. Indeed, the experimental results highlighted the simulation trend of an excessive sensitivity of the PI-FGS controller. This problem was solved by imposing a transition time of $0.5 \mathrm{~s}$ between a ramp and a plateau (or conversely between a plateau and a ramp). This turns out to be negligible in a regular test, but very useful to help the controller in avoiding excessive overshooting. Thus, a new reference signal is built by calculating the acceleration $(A c c)$ and deceleration (Dec) of the ramp-plateau transition according to the ramp rate $(r r): 25,26$

$$
\operatorname{Acc}(D e c)=(-) r r / \Delta t,
$$

where $\Delta t$ is $0.5 \mathrm{~s}$.

In Fig. 10(a), an example of the measured current $\left(I^{*}{ }_{m}\right)$ using the PI and the PI-FGS control strategies is shown. The main parameters of the reference current $\left(I^{*}\right.$ ref $)$ for this cycle are: $I_{\max }=24.5 \mathrm{kA}, r r=800 \mathrm{~A} / \mathrm{s}$, and $A c c=D e c$ $=1600 \mathrm{~A} / \mathrm{s}^{2}$.

In Fig. 10(b1) the magnification of the plateau phase is illustrated: the smaller overshoot $(10 \mathrm{~A})$ of the PIFGS can be appreciated, that is, larger bandwidth. In Fig. 10(b2), the difference between the measured current $\left(I^{*}{ }_{\mathrm{m}}\right)$ and the ideal reference $\left(I_{\text {ref }}^{*}\right)$ for both control algorithms is depicted.

A view of the tracking error, expressed as RMSE, obtained with the PI strategy and the PI-FGS system is given in Table I as a function of maximum current and ramp rate; the

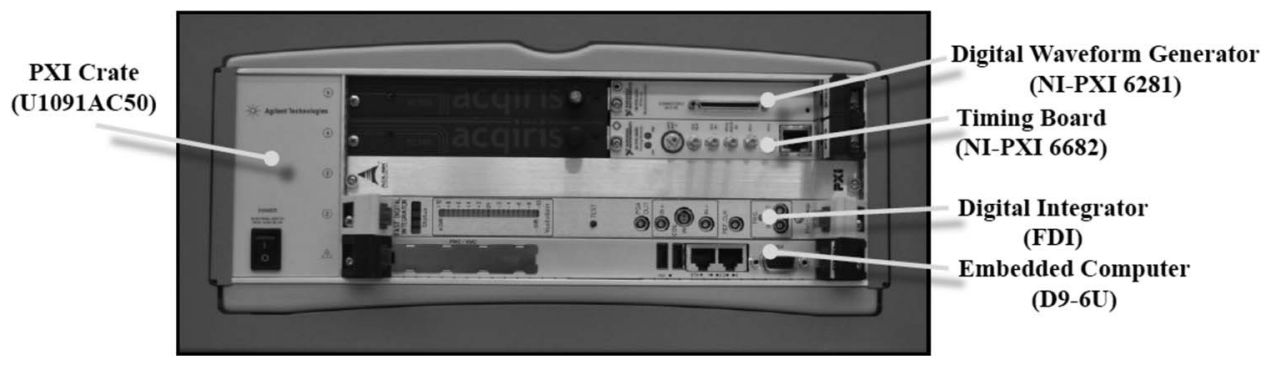

FIG. 9. Measurement and control instrumentation setup. ${ }^{1}$ 

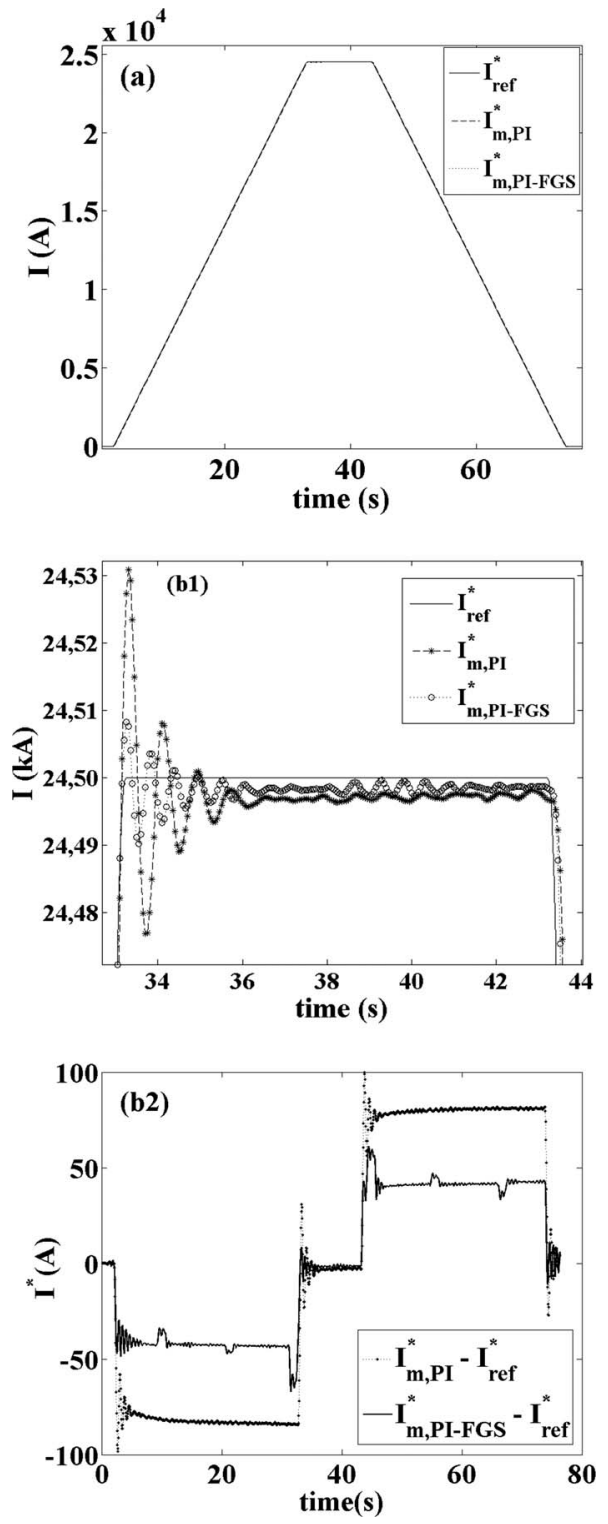

FIG. 10. Measured currents using the PI $\left(I^{*}{ }_{\mathrm{m}, \mathrm{PI}}\right)$ and the PI-FGS $\left(I_{\mathrm{m}, \mathrm{PI}-\mathrm{FGS}}\right)$ control algorithms for the reference $I^{*}{ }_{\text {ref }}$ current cycle at $24.5 \mathrm{kA}$ (a) and magnification of the measured currents $I^{*}{ }_{\mathrm{m}, \mathrm{PI}}$ and $I^{*}{ }_{\mathrm{m}, \mathrm{PI}-\mathrm{FGS}}$ during the phase of plateau (b1); differences among the measured currents $I^{*}{ }_{\mathrm{m}, \mathrm{PI}}$ and $I_{\mathrm{m}, \mathrm{PI}-\mathrm{FGS}}^{*}$ (b2) and the reference cycle $\left(I^{*}{ }_{\text {ref }}\right)$, the current function that has to be induced in the cable under test, complete the picture.

acceleration and deceleration values are calculated according to (4.1). In this configuration, the overshooting phenomenon is still more evident at higher ramp rates than at lower currents.

The absolute overshooting value is almost constant for the same ramp rate, thus the relative value decrease drastically with increasing current values. The collected results show a common trend, regardless the considered ramp rate and maximum current. Under the same conditions, the error for the adaptive control strategy is considerably less, sometimes by more than $50 \%$, with respect to the classical control strategy. Such an error is accumulated mainly in the ramp phases; under these circumstances, the PI-FGS controller shows satisfactory promptness in following the ramp and negligible oscillations during transitions between ramp and plateau.
TABLE I. Aggregated RMSE mean values (in kA) from the PI-FGS/PI comparison.

\begin{tabular}{|c|c|c|c|c|c|c|c|c|}
\hline \multirow{2}{*}{$\begin{array}{l}\text { Ramp Rate } \\
I_{\max }(\mathrm{kA})\end{array}$} & \multicolumn{2}{|c|}{$800(\mathrm{~A} / \mathrm{s})$} & \multicolumn{2}{|c|}{$\overline{500(\mathrm{~A} / \mathrm{s})}$} & \multicolumn{2}{|c|}{$300(\mathrm{~A} / \mathrm{s})$} & \multicolumn{2}{|c|}{$100(\mathrm{~A} / \mathrm{s})$} \\
\hline & FGS & PI & FGS & PI & FGS & PI & FGS & PI \\
\hline 5.00 & 25.52 & 48.36 & 18.24 & 34.28 & 14.15 & 22.99 & 4.74 & 8.85 \\
\hline 10.00 & 30.31 & 58.69 & 20.77 & 40.10 & 13.41 & 25.92 & 4.98 & 9.52 \\
\hline 15.00 & 33.08 & 64.03 & 22.24 & 42.96 & 14.13 & 27.29 & 5.14 & 9.86 \\
\hline 20.00 & 34.93 & 67.38 & 23.20 & 44.72 & 14.62 & 28.15 & 5.28 & 10.11 \\
\hline 24.50 & 36.60 & 69.52 & 24.15 & 45.83 & 15.15 & 28.72 & 5.43 & 10.29 \\
\hline
\end{tabular}

The RMSE reduction of almost a factor of 2, when using the novel FGS control as compare to previous PI control, is evident from the data in Table I.

\section{Experimental validation}

The performance of the PI-FGS control strategy against the reference PI-based system was validated characterizing the same LHC outer layer dipole cable (LHC cable of type 2) used for the previous PI system validation. ${ }^{1}$ Main goal of the comparative tests is to prove that PI-FGS does not looses in metrological performance against a reduction in overshoot of about 50\% (Fig. 10). At this aim, tests have to prove that the PI-FGS and PI state-the-art algorithm have similar repeatability. "Similarity" is assessed in terms of reproducibility of the results versus a change in measurement algorithm. In other terms, according to the 3rd edition of the International Vocabulary of Metrology (VIM3), ${ }^{27}$ the reproducibility conditions under test is the measurement algorithm change from PI to PI-FGS. For this reason, comparative tests aim to show that the repeatability value is compatible with the state-of-the-art reproducibility.

A measurement campaign, with background field from 3.0 to $9.0 \mathrm{~T}$, and current ramp rates from 50 to $800 \mathrm{~A} / \mathrm{s}$, was carried out by switching the control software between the PI and PI-FGS algorithms, respectively. The values of the measured critical current for the sample cable under test are reported in Table II, both with the reference (PI) algorithm and with the novel (adaptive PI-FGS) controller. This type of measurement has an expected repeatability of $\pm 0.5 \%$ and reproducibility of $\pm 2 \% .^{3}$ In the comparative tests, the repeatability was assessed by computing the experimental dispersion of the critical current in several measurements for the same imposed external field. In particular, the repeatability of the reference critical current $I_{\mathrm{c}, \mathrm{PI}}$ was better than $\pm 0.6 \%{ }^{1}$

The set of measurements for the PI-FGS algorithm in Table II shows repeatability of the order of $\pm 0.5 \%$. Moreover, the maximum error among the measured critical

TABLE II. Average critical current values using the PI and PI-FGS algorithms.

\begin{tabular}{lrrr}
\hline \hline Applied field (T) & $I_{\mathrm{c}, \mathrm{PI}}(\mathrm{A})$ & $I_{\mathrm{c}, \mathrm{PI}-\mathrm{FGS}}(\mathrm{A})$ & Error $(\%)$ \\
\hline 3 & 21980 & $22287 \pm 0.061$ & 1.96 \\
5 & 15255 & $15361 \pm 0.081$ & -1.97 \\
7 & 8737 & $8568 \pm 0.045$ & 0.70 \\
9 & 2553 & $2604 \pm 0.025$ & 1.34 \\
\hline \hline
\end{tabular}




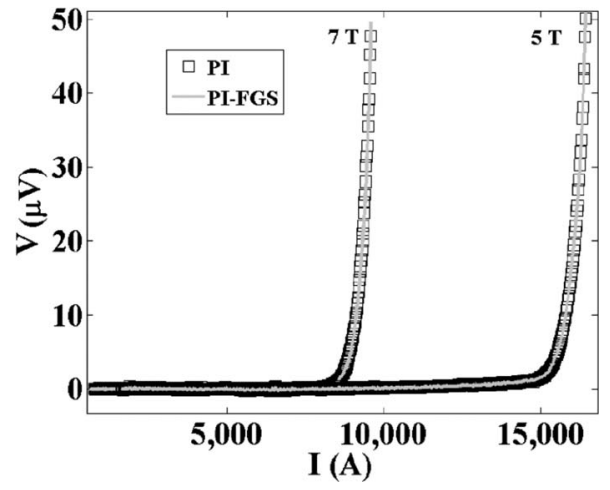

FIG. 11. Comparisons of V-I curves on a LHC cable of type 2, measured using the reference PI control algorithm and the novel PI-FGS system. In the two experimental curves displayed, the current ramp rate is $50 \mathrm{~A} / \mathrm{s}$ at $7 \mathrm{~T}$ and $250 \mathrm{~A} / \mathrm{s}$ at $5 \mathrm{~T}$, respectively.

current values is of the order of $\pm 2 \%$, most likely arising from the improvement in the ramp up phase introduced by the PIFGS. Therefore, the worst-case reproducibility is of the order of $\pm 2 \%$ compatible with the above referenced literature value usual in this application.

As an example, in Fig. 11, the measured V-I curves on a $610 \mathrm{~mm}$ long cable are compared at $5.0 \mathrm{~T}$ and $7.0 \mathrm{~T}$, with current ramp rate of $250 \mathrm{~A} / \mathrm{s}$ and $50 \mathrm{~A} / \mathrm{s}$, respectively. The measured voltages with the two controllers do overlap significantly and the noise level is well within the requirements (below $2 \mu \mathrm{V}$ ). The differences in terms of measured critical current values cannot be appreciated at a glance, even if a zoom-in is performed on the graph, because the values of $I_{\mathrm{c}, \mathrm{PI}}$ and $I_{\mathrm{c}, \mathrm{PI}-\mathrm{FGS}}$ are close to each other and their measure is obtained as the result of a fitting process on the experimental data.

\section{CONCLUSIONS}

At the Facility for the Research of Superconducting Cables $^{3}$ (FReSCa) of the European Organization for Nuclear Research (CERN), a measurement system based on a superconducting transformer is exploited. ${ }^{1}$ Metrological performance of the control driving the current into the cable under test has been improved by an adaptive control strategy. ${ }^{8}$ This system proved to be effective in overcoming the limitations shown by the previously available measurement system by means of a faster and more reliable control of the current in the transformer secondary.

The current control strategy was designed by following the Gain Scheduling logic, ${ }^{9}$ thus varying the controller parameters according to changes in the working condition. The variations in the controller are driven by the reference current $I_{\text {ref }}$, whose domain was divided into subspaces according to the system behaviour. By identifying each local domain dynamic, a model of the chain composed by the current source and the transformer was synthesized and the subspaces obtained were linked to each other by means of Fuzzy Logic. ${ }^{13}$ The chosen system input was the voltage reference $V_{\text {ref }}$, driving the current source, while the secondary current was taken as the system output. The scheduling function that allows navigating among the different subdomains was implemented again through the Fuzzy Logic. ${ }^{16}$ Thus, a PI-FGS system was designed by tuning the local PI controllers, one associated to each subdomain, and implementing each controller in the consequent of the TSK-type inference system ${ }^{16}$ chosen for the FGS development.

The effectiveness of the novel architecture was assessed by an experimental implementation aimed at controlling the superconducting transformer available at the FReSCa of CERN.

Achieved key performance improvements are

- short response overshoot for the most critical current and ramp rates, keeping the same capability to follow the reference ramp as the previous control system;

- reduction in the error (both absolute and RMS) up to $52 \%$ in the compared results with respect to the state-of-the-art-control strategy previously adopted at CERN.

The improvement achieved in the ramp phase, given by the reduction in the delay introduced by the PI-FGS strategy, together with the ability to settle within the given transition time, are the main reasons for the significant error decreasing. This improvement turned into the possibility to run cycle with acceleration up to $1600 \mathrm{~A} / \mathrm{s}^{2}$ for an $800 \mathrm{~A} / \mathrm{s}^{1}$ ramp rate.

These results were demonstrated in practical working conditions, measuring the critical current of a NbTi Rutherford cable with well-known properties. ${ }^{1}$ Measurements of the critical current show full compatibility of the PI-FGS-based system with the available reference at FReSCa, i.e., the PIbased system. The comparison of the proposed smart controller system with the available reference highlights reproducibility better than $2 \%$, which is the typical requirement of the critical current for the cables under test.

\section{ACKNOWLEDGMENTS}

This work was supported by CERN through the agreement TE/K1776 with the University of Sannio, whose support the authors acknowledge gratefully. The authors also thank L. Bottura, F. Cennamo, G. Peiro, L. Fiscarelli, and G. Willering for their contribution, useful suggestions and cooperation.

${ }^{1}$ P. Arpaia, L. Bottura, G. Montenero, and S. Le Naour, "Performance improvement of a measurement station for superconducting cable test," Rev. Sci. Instrum. 83, 095111 (2012).

${ }^{2}$ W. Buckel and R. Kleiner, Superconductivity Fundamentals and Applications (WILEY-VCH Verlag GmbH \& Co. KGaA, Weinheim, 2004), pp. 269-302.

${ }^{3}$ A. P. Verweij and A. K. Ghosh, "Critical current measurements of the Main LHC superconducting cables,” IEEE Trans. Appl. Supercond. 17(2), 1454 (2007).

${ }^{4}$ A. P. Verweij, J. Genest, A. Knezovic, D. F. Leroy, J.-P. Marzolf, and L. R. Oberli, "1.9 K test facility for the reception of the superconducting cables for the LHC," IEEE Trans. Appl. Supercond. 9(2), 153 (1999).

${ }^{5}$ E. Barzi, N. Andreev, J. Brandt, C. Carmignani, M. Danuso, V. V. Kashikhin, V. S. Kashikhin, S. Mintchev, D. Mitchell, D. Turrioni, A. Rusy, and A. V. Zlobin, "Superconducting transformer for superconducting cable tests in a magnetic field," AIP Conf. Proc. 1218, 421-428 (2010).

${ }^{6}$ H. J. Liu, Y. Wu, Zh. B. Ren, S. T. Wu, Y. Shi, J. Q. Peng, J. L. Chen, F. Long, M. Yu, and L. Qian, "Manufacturing of $50 \mathrm{kA}$ superconducting transformer for ITER correction coil conductor test,” Rev. Sci. Instrum. 81, 044701 (2010). 
${ }^{7}$ A. Godeke, D. R. Dietderich, J. M. Joseph, J. Lizarazo, S. O. Prestemon, G. Miller, and H. W. Weijers, "A superconducting transformer system for high current cable testing," Rev. Sci. Instrum. 81, 035107 (2010) .

${ }^{8}$ M. Krstić, I. Kannellakopoulos, and P. Kokotović, Nonlinear and Adaptive Control Design (Wiley, London, 1995).

${ }^{9}$ J.-T. Su and C.-W. Liu, "Gain scheduling control scheme for improved transient response of DC/DC converters," IET Power Electron. 5, 678 (2012).

${ }^{10} \mathrm{R}$. Garduno-Ramirez and K. Y. Lee, "Fuzzy scheduling control of a power plant," Power Engineering Society Winter Meeting (IEEE, 2000), Vol. 1, pp. $441-445$.

${ }^{11}$ D. J. Leith and W. E. Leithead, "Survey of gain-scheduling analysis and design," Int. J. Control 73, 1001 (2010).

${ }^{12}$ K. M. Passino and S. I. Yurkovich, Fuzzy Control (Addison Wesley Longman, Inc., 1998).

${ }^{13}$ T. Takagi and M. Sugeno, "Fuzzy identification of systems and its applications to modeling and control," IEEE Trans. Syst., Man, Cybern. SMC15(1), 116-132 (1985).

${ }^{14}$ O. Nelles, Nonlinear System Identification (Springer, 2001), Vol. XVII, p. 785.

${ }^{15}$ A. Rodriguez-Martinez, R. Garduno-Ramirez, and L. G. VelaValdes, "PI fuzzy gain-scheduling speed control at startup of a gasturbine power plant," IEEE Trans. Energy Convers. 26(1), 310-317 (2011).

${ }^{16}$ See http://sine.ni.com/nips/cds/view/p/lang/en/nid/14119 for NI-PXI 6281; accessed 12 November 2011

${ }^{17}$ T. Boutboul, C. H. Denarié, Z. Charifoulline, L. Oberli, and D. Richter, "Critical Current Test Facilities for LHC Superconducting NbTi Cable
Strands," LHC Project Report 520 (CERN, Geneva, Switzerland, 2001), p. 8 .

${ }^{18}$ See www.lakeshore.com/ObsoleteAndResearchDocs/MPS_Manual.pdf for Model 620/622/647; accessed 12 November 2011

${ }^{19}$ P. Arpaia, L. Bottura, L. Fiscarelli, and L. Walckiers, "Performance of a fast digital integrator in on-field magnetic measurements for particle accelerators," Rev. Sci. Instrum. 83, 024702 (2012).

${ }^{20}$ See http://digital.ni.com/manuals.nsf/websearch/6113304BCA14E823862 5757E00691D26? OpenDocument\&seen=1 for NI PXI-6682; accessed 12 November 2011.

${ }^{21}$ See http://cp.literature.agilent.com/litweb/pdf/U1092-90018.pdf for Agilent Acqiris 3-, 5-, and 8-Slot cPCI Crates; accessed 12 November 2011.

${ }^{22}$ See http://www.men.de/support/downloads/w2=D9\&t=doc,sw.html for D9 - 6U; accessed 12 November 2011.

${ }^{23}$ P. Arpaia, M. Buzio, L. Fiscarelli, and V. Inglese, "A software framework for developing measurement applications under variable requirements," Rev. Sci. Instrum. 83, 115103 (2012).

${ }^{24}$ T. Shimada, M. Sugimoto, Y. Nagasu, A. Takagi, K. Wada, H. Shimizu, A. Kimura, and S. Meguro, "Manufacturing of superconducting cable for the LHC-key technology and statistical analysis," IEEE Trans. Appl. Supercond. 12(1), 1075 (2002).

${ }^{25}$ H. H. J. ten Kate, J. M. Mulders, J. L. de Reuver, and L. J. M. van de Klundert, "Ac loss measurements on a superconducting transformer for a 25 kA superconducting rectifier," Cryogenics 24(8), 439 (1984).

${ }^{26}$ J. L. de Reuver, H. H. J. ten Kate, H. G. Knoopers, and L. J. M. van de Klundert, "Dc and ac behaviour of a normal joint in a superconducting cable," Cryogenics 24(5), 251 (1984).

${ }^{27}$ International Vocabulary of Metrology - Basic and General Concepts and Associated Terms (VIM), 3rd ed. (JCGM, 2012), p. 200. 